JOURNAL OF

FUNCTION SPACES AND APPLICATIONS

Volume 8, Number 2 (2010), 103-128
(C) 2010, Scientific Horizon http://www.jfsa.net

\title{
Lipschitz estimates for the Berezin transform
}

\author{
Hélène Bommier-Hato
}

(Communicated by Miroslav Englǐs)

2000 Mathematics Subject Classification. 47B35, 32A35, 32A25.

Keywords and phrases. Berezin transform.

\begin{abstract}
We consider the generalized Fock space $\mathcal{A}^{2}\left(\mu_{m}\right)$, where $\mu_{m}$ is the measure with weight $e^{-|z|^{m}}, m>0$, with respect to the Lebesgue measure on $\mathbb{C}^{n}$. Improving upon a recent result of L. Coburn and J. Xia, we show that for any bounded operator $X$ on $\mathcal{A}^{2}\left(\mu_{m}\right)$, the Berezin transform of $X$ satisfies Lipschitz estimates.
\end{abstract}

\section{Introduction and statement of the main results}

We consider the Fock space $\mathcal{A}^{2}\left(\mu_{m}\right)$ of those holomorphic functions which are square integrable on $\mathbb{C}^{n}$ with respect to the measure $d \mu_{m}(z)=$ $e^{-|z|^{m}} d V(z)$, where $d V(z)$ is the Lebesgue measure and $m>0$ is a positive parameter. Let $K_{m}$ be the Bergman kernel of the Hilbert space $\mathcal{A}^{2}\left(\mu_{m}\right)$ and denote by $k_{a}$ the corresponding normalized kernel. It is given by

$$
k_{a}(z):=K(z, a) / \sqrt{K(a, a)}
$$

and is a unit vector of $\mathcal{A}^{2}\left(\mu_{m}\right)$. The Berezin transform of a bounded linear operator $X$ on $\mathcal{A}^{2}\left(\mu_{m}\right)$ is the function defined by $\widetilde{X}(a)=\left\langle X k_{a}, k_{a}\right\rangle$.

Recently, Coburn [4], [5] has shown that in the classical case $m=2$, 
corresponding to the Segal-Bargmann space endowed with the Gaussian measure on $\mathbb{C}^{n}, \widetilde{X}$ is a Lipschitz function, satisfying

$$
|\widetilde{X}(a)-\widetilde{X}(b)| \leq 2\|X\||a-b|
$$

where $\|X\|$ is the norm of $X$.

The main goal of this paper is to investigate the general case of positive parameters $m>0$. Namely, we will show that the Berezin transform is locally Lipschitz and when $n=1$ and $m \leq 1$, the Lipschitz estimate is uniform. In the proof of our results we also establish estimates of the quantity

$$
\rho_{m}(a, b):=\sqrt{1-\left|\left\langle k_{a}, k_{b}\right\rangle\right|^{2}} .
$$

If $A$ and $B$ are two quantities, we use the symbol $A \approx B$ whenever $A \leq C_{1} B$ and $B \leq C_{2} A$, where $C_{1}$ and $C_{2}$ are positive constants independent of the varying parameters.

Our main result is the following

Theorem 1. If $X$ is a bounded operator on $\mathcal{A}^{2}\left(\mu_{m}\right)$, then its Berezin transform $\widetilde{X}$ is locally Lipschitz. In addition, there exist positive constants $A, C$, depending on $n$ and $m$ only, such that for any $a \in \mathbb{C}^{n}$ with $|a|>A$, there is a neighbourhood $U$ of a that satisfies

$$
|\tilde{X}(x)-\tilde{X}(a)| \leq C\|X\||a|^{\frac{m}{2}-1}|x-a|, \text { for } x \in U .
$$

The proof of Theorem 1 gives a more accurate estimate of $\rho_{m}(a, b)$ when $n=1$.

Theorem 2. On $\mathbb{C}$, the quantity $\rho_{m}(a, b)$ satisfies a Lipschitz estimate. More precisely, there exists a positive constant $A$, depending on $m$ only, such that for any $a \in \mathbb{C}^{n}$ with $|a|>A$, there is a neighbourhood $U$ of a that satisfies

$$
\rho_{m}(a, x) \approx|a|^{\frac{m}{2}-1}|x-a|, \text { for } x \in U .
$$

When $\mathrm{m}$ is an even integer, we have the following consequence of Theorem 2:

Corollary 3. Suppose $n=1$ and $m$ an even positive integer. There exists a positive constant $A$, depending on $n$ and $m$ only, such that, for any $a \in \mathbb{C}$ with $|a|>A$, there is a neighbourhood $U$ of a that satisfies

$$
\rho_{m}(a, x) \approx\left|a^{m / 2}-x^{m / 2}\right| \text { for all } x \in U .
$$


A careful study of $\rho_{m}(a, x)$ when $m \leq 1$ shows that the Lipschitz condition is uniform.

Corollary 4. Assume $n=1$ and $m \leq 1$ and let $X$ be a bounded operator on $\mathcal{A}^{2}\left(\mu_{m}\right)$. Then $\widetilde{X}$ is Lipschitz. More precisely, there are positive constants $A, C, \delta$, depending on $n$ and $m$ only, such that for any $a \in \mathbb{C}^{n}$ with $|a|>A$,

$$
|\widetilde{X}(x)-\tilde{X}(a)| \leq C\|X\||x-a|, \text { for all } x \text { with }|x-a|<\delta .
$$

\section{Preparatory results}

We will use a theorem shown by Coburn in [4], which is valid for any reproducing kernel.

Lemma 1. For a bounded operator $X$ on $\mathcal{A}^{2}\left(\mu_{m}\right)$ and $a, b$ in $\mathbb{C}^{n}$,

$$
|\tilde{X}(b)-\tilde{X}(a)| \leq 2\|X\| \rho_{m}(a, b) .
$$

We recall

$$
\rho_{m}(a, b)^{2}=\frac{1}{K_{m}(a, a) K_{m}(b, b)}\left[K_{m}(a, a) K_{m}(b, b)-\left|K_{m}(a, b)\right|^{2}\right],
$$

where $K_{m}$ is the Bergman kernel of $\mathcal{A}^{2}\left(\mu_{m}\right)$. It follows from [1] that the Bergman kernel $K_{m}$ can be expressed in terms of the generalized MittagLeffler function

$$
E_{\frac{2}{m}, \frac{2}{m}}(\lambda):=\sum_{d=0}^{+\infty} \frac{\lambda^{d}}{\Gamma\left(\frac{2 d}{m}+\frac{2}{m}\right)}
$$

by the equation

$$
K_{m}(z, w)=\frac{m}{(n-1) !} E_{\frac{2}{m}, \frac{2}{m}}^{(n-1)}(\langle z, w\rangle)
$$

Setting $\alpha:=2 / m$ and $F:=E_{\alpha, \alpha}^{(n-1)}$, we see that for $a, u$ in $\mathbb{C}^{n}$,

$\rho_{m}(a, a+u)^{2}=\frac{1}{F\left(|a|^{2}\right) F\left(|a+u|^{2}\right)}\left\{F\left(|a|^{2}\right) F\left(|a+u|^{2}\right)-|F(\langle a, a+u\rangle)|^{2}\right\}$.

In order to study $\rho_{m}(a, a+u)^{2}$ near $a$, we will use a Taylor's expansion formula.Thus we need to estimate $F$ and its higher order derivatives. 


\section{Asymptotic expansion of the derivatives of the Mittag-Leffler function $E_{\alpha, \alpha}$}

Following the method developped by Wong and Zhao in [10], we consider several subcases, corresponding to different values of $\alpha$.

Assume $p$ is a non-negative integer. For $z \in \mathbb{C} \backslash\{0\}$, we suppose the branch of the argument is chosen such that $\arg z \in[-\pi, \pi[$. If $s$ is an integer, let

$$
Z_{s}:=|z|^{\frac{1}{\alpha}} \exp \left[\frac{i}{\alpha}(\arg z+2 \pi s)\right]
$$

and denote by $\mathcal{S}$ the set of those integers $s$ satisfying $\left|\arg Z_{s}\right|<\pi$.

Lemma 2. If $\alpha \in \mathbb{N}_{0} \backslash\{0\}$, then there exists a polynomial $P_{p+1}$ of degree $p+1$ such that for any $z \neq 0$,

$$
E_{\alpha, \alpha}^{(p)}(z)=\sum_{s=0}^{\alpha-1} e^{Z_{s}} Z_{s}^{-\alpha(p+1)} P_{p+1}\left(Z_{s}\right) .
$$

Proof. We recall the Hankel representation of the entire function $1 / \Gamma$

$$
\frac{1}{\Gamma(w)}=\frac{1}{2 \pi i} \int_{\gamma_{a, \phi_{0}}} e^{t} t^{-w} d t
$$

where $\frac{\pi}{2}<\phi_{0}<\pi,|z|<a^{\alpha}$ and $\gamma_{a, \phi_{0}}$ is the Hankel contour which starts at $\infty e^{-i \phi_{0}}$, follows the half-ray $\arg t=-\phi_{0}$, encircles the origin once counterclockwise on the circular part $|t|=a$ and returns to $\infty e^{i \phi_{0}}$ on the half-ray $\arg t=\phi_{0}$.

Fix $z \in \mathbb{C} \backslash\{0\}$, and if $d$ is an integer, set $I_{d}:=\frac{z^{d}}{\Gamma(\alpha d+\alpha)}$. Then from the formula above we have

$$
I_{d}=\frac{1}{2 \pi i} \int_{\gamma_{a, \phi_{0}}} e^{t} t^{-\alpha}\left(\frac{z}{t^{\alpha}}\right)^{d} d t .
$$

We write $I_{d}=I_{d, \text { lin }}+I_{d, \text { circ }}$, where $I_{d, \text { lin }}$ and $I_{d, \text { circ }}$ are respectively the integrals on the linear part and circular part of $\gamma_{a, \phi_{0}}$.

On the half rays of $\gamma_{a, \phi_{0}}$ we have

$$
\left|I_{d, l i n}\right| \leq 2 \frac{1}{2 \pi} \int_{a}^{+\infty} e^{\tau \cos \phi_{0}} \tau^{-\alpha}\left|\frac{z}{\tau^{\alpha}}\right|^{d} d \tau
$$


Therefore

$$
\sum_{d=0}^{+\infty}\left|I_{d, l i n}\right| \leq \frac{1}{\pi} \int_{a}^{+\infty} e^{\tau \cos \phi_{0}} \tau^{-\alpha} \frac{1}{1-\frac{|z|}{\tau^{\alpha}}} d \tau=\frac{1}{\pi} \int_{a}^{+\infty} \frac{e^{\tau \cos \phi_{0}}}{\tau^{\alpha}-|z|} d \tau<+\infty .
$$

On the circular part of $\gamma_{a, \phi_{0}}$ we have

$$
\left|I_{d, c i r c}\right| \leq \frac{1}{2 \pi} \int_{-\phi_{0}}^{\phi_{0}} e^{a \cos \phi} a^{-\alpha}\left(\frac{|z|}{a^{\alpha}}\right)^{d} d \phi
$$

Then

$$
\sum_{d=0}^{+\infty}\left|I_{d, c i r c}\right| \leq 2 \frac{1}{2 \pi} \phi_{0} e^{a \cos \phi_{0}} \frac{1}{a^{\alpha}-|z|} .
$$

Thus we can write

$$
E_{\alpha, \alpha}(z)=\frac{1}{2 \pi i} \int_{\gamma_{a, \phi_{0}}} e^{t} t^{-\alpha} \sum_{d=0}^{+\infty}\left(\frac{z}{t^{\alpha}}\right)^{d} d t=\frac{1}{2 \pi i} \int_{\gamma_{a, \phi_{0}}} e^{t} \frac{d t}{t^{\alpha}-z} .
$$

Let $p$ be a non-negative integer. On the linear part of $\gamma_{a, \phi_{0}}, t=$ $\tau e^{ \pm i \phi_{0}}$ with $\tau \geq a$, then $\left|\frac{e^{t}}{\left(t^{\alpha}-z\right)^{p+1}}\right| \leq \frac{e^{\tau \cos \phi_{0}}}{\left(\tau^{\alpha}-|z|\right)^{p+1}}$, and the integral $\int_{a}^{+\infty} \frac{e^{\tau \cos \phi_{0}}}{\left(\tau^{\alpha}-z\right)^{p+1}} d t$ is convergent.

By differentiation we get

$$
E_{\alpha, \alpha}^{(p)}(z)=\frac{1}{2 \pi i} \int_{\gamma_{a, \phi_{0}}} f_{p}(t) d t
$$

where

$$
f_{p}(t):=p ! \frac{e^{t}}{\left(t^{\alpha}-z\right)^{p+1}}
$$

Now we will get another expression of this integral by deforming the contour $\gamma_{a, \phi_{0}}$. Choose $r, R, \phi_{1}$ such that $0<r<a<R, r^{\alpha}<|z|<a^{\alpha}$, and $0<\phi_{1}<\pi-\phi_{0}$. Consider the counter-clockwise loop which starts at $R e^{i\left(-\pi+\phi_{1}\right)}$, follows the circular path $|t|=R$ to $R e^{-i \phi_{0}}$, a straight line to $a e^{-i \phi_{0}}$, a part of the circle $|t|=a$ to $a e^{i \phi_{0}}$, a straight line to $R e^{i \phi_{0}}$, the circle $|t|=R$ to $R e^{i\left(\pi-\phi_{1}\right)}$, a straight horizontal line to $r e^{i \frac{\pi}{2}}$, the semicircle $|t|=r$ to $r e^{-i \frac{\pi}{2}}$, then a straight horizontal line to the starting point $R e^{i\left(-\pi+\phi_{1}\right)}$.

For any integer $s$ we put

$$
Z_{s}:=|z|^{\frac{1}{\alpha}} \exp \left[\frac{i}{\alpha}(\arg z+2 \pi s)\right]
$$


and

$$
\theta_{s}:=\frac{1}{\alpha}(\arg z+2 \pi s) .
$$

Define the set $\mathcal{S}:=\left\{s \in \mathbb{Z},\left|\arg Z_{s}\right|<\pi\right\}$. If we choose $r$ small enough for the poles $Z_{s}$ (such that $\left|\arg Z_{s}\right|<\pi$ ) of $f_{p}(t)$ to be inside $\gamma_{a, \phi_{0}, R, r}$, the theorem of residues leads to

$$
\frac{p !}{2 \pi i} \int_{\gamma_{a, \phi_{0}, R, r}} e^{t} \frac{d t}{\left(t^{\alpha}-z\right)^{p+1}}=\sum_{s \in \mathcal{S}} \operatorname{Res}\left(f_{p} ; Z_{s}\right) .
$$

On the big circular part of $\gamma_{a, \phi_{0}, R, r}$, we have

$$
\left|\int_{\phi_{0}}^{\pi-\phi_{1}} \frac{e^{R e^{i \phi}}}{\left(R^{\alpha} e^{i \alpha \phi}-z\right)^{p+1}} i e^{i \phi} R d \phi\right| \leq \pi R \frac{e^{R \cos \phi_{0}}}{\left(R^{\alpha}-|z|\right)^{p+1}}
$$

and

$$
\left|\int_{-\pi+\phi_{1}}^{-\phi_{0}} \frac{e^{R e^{i \phi}}}{\left(R^{\alpha} e^{i \alpha \phi}-z\right)^{p+1}} i e^{i \phi} R d \phi\right| \leq \pi R \frac{e^{R \cos \phi_{0}}}{\left(R^{\alpha}-|z|\right)^{p+1}}
$$

Since $\cos \phi_{0}<0$, we see that

$$
\lim _{R \rightarrow+\infty} \frac{R e^{R \cos \phi_{0}}}{\left(R^{\alpha}-|z|\right)^{p+1}}=0 .
$$

When $R$ tends to $+\infty, \gamma_{a, \phi_{0}, R, r}$ is deformed in two paths, namely $\gamma_{a, \phi_{0}}$ and $\gamma_{r}^{\prime}$, where $\gamma_{r}^{\prime}$ starts at $-i r-\infty$, follows a straight horizontal line to $r e^{-i \frac{\pi}{2}}$, encirles the origin once counter-clockwise on the semi-circle $|t|=r$, and follows a straight horizontal line to $i r-\infty$.

On the rectilinear part of $\gamma_{r}^{\prime}$ we have $\left|\frac{e^{t}}{\left(t^{\alpha}-z\right)^{p+1}}\right| \leq \frac{e^{\tau \cos \phi_{0}}}{\left(|\tau|^{\alpha}-|z|\right)^{p+1}} \mid$, thus the integral $\int_{\gamma_{r}^{\prime}} e^{t} \frac{d t}{\left(t^{\alpha}-z\right)^{p+1}}$ is absolutely convergent .

Therefore

$$
\sum_{s \in \mathcal{S}} \operatorname{Res}\left(f_{p} ; Z_{s}\right)=\frac{p !}{2 \pi i} \int_{\gamma_{a, \phi_{0}}} e^{t} \frac{d t}{\left(t^{\alpha}-z\right)^{p+1}}-\frac{p !}{2 \pi i} \int_{\gamma_{r}^{\prime}} e^{t} \frac{d t}{\left(t^{\alpha}-z\right)^{p+1}},
$$

so that

$$
E_{\alpha, \alpha}^{(p)}(z)=\sum_{s \in \mathcal{S}} \operatorname{Res}\left(f_{p} ; Z_{s}\right)+\frac{1}{2 \pi i} \int_{\gamma_{r}^{\prime}} f_{p}(t) d t .
$$

Next, for $s \in \mathcal{S}$, we shall compute $\operatorname{Res}\left(f_{p} ; Z_{s}\right)$. Let $\mathcal{C}_{s}$ be a circle centered at $Z_{s}$ of radius $\eta$ and $\mathcal{C}_{s}^{\prime}$ the image of $\mathcal{C}_{s}$ by the function power $\alpha$, where we choose $\eta$ small enough for $\mathcal{C}_{s}$ and $\mathcal{C}_{s}^{\prime}$ to be included in $\mathbb{C} \backslash \mathbb{R}_{-}$ and the function power $\alpha$ is determined by $u=t^{\alpha}=\exp [\alpha|\ln t|+$ 
$i \alpha \arg t],|\arg t|<\pi$. In a neighbourhood of $\mathcal{C}_{s}^{\prime}$, the function power $\frac{1}{\alpha}$ is defined by

$$
u^{\frac{1}{\alpha}}=\exp \left[\frac{1}{\alpha} \ln |u|+\frac{i}{\alpha}(\arg u+2 \pi s)\right],|\arg u|<\pi .
$$

The change of variables $u=t^{\alpha}$ shows that

$$
\operatorname{Res}\left(f_{p} ; Z_{s}\right)=\frac{1}{2 \pi i} p ! \int_{\mathcal{C}_{s}^{\prime}} \frac{e^{u}}{(u-z)^{p+1}} \frac{1}{\alpha} u^{\frac{1}{\alpha}-1} d u .
$$

We get $\operatorname{Res}\left(f_{p} ; Z_{s}\right)=\frac{d^{p+1}}{d u^{p+1}}\left[e^{u^{\frac{1}{\alpha}}}\right]_{u=z}$.

Now, we have to compute the higher order derivatives of $h(u):=e^{u^{\frac{1}{\alpha}}}$. By induction we see that, for any $p \in \mathbb{N}_{0}$, there exists a polynomial $P_{p}$ of degree $p$ such that $h^{(p)}(u)=\frac{e^{u^{\frac{1}{\alpha}}}}{u^{p}} P_{p}\left(u^{\frac{1}{\alpha}}\right)$. By differentiating the last equality, we get

$$
h^{(p+1)}(u)=\left[\frac{e^{u^{\frac{1}{\alpha}}}}{u^{p}} P_{p}\left(u^{\frac{1}{\alpha}}\right)\right]^{\prime}=\frac{e^{u^{\frac{1}{\alpha}}}}{u^{p+1}}\left[\frac{1}{\alpha} u^{\frac{1}{\alpha}} P_{p}\left(u^{\frac{1}{\alpha}}\right)+\frac{1}{\alpha} u^{\frac{1}{\alpha}} P_{p}^{\prime}\left(u^{\frac{1}{\alpha}}\right)-p P_{p}\left(u^{\frac{1}{\alpha}}\right)\right] .
$$

It follows that the polynomials $\left(P_{p}\right)_{p \in \mathbb{N}}$ satisfy the relation:

$$
P_{p+1}(t)=\frac{1}{\alpha} t P_{p}(t)+\frac{1}{\alpha} t P_{p}^{\prime}(t)-p P_{p}(t) .
$$

Since $P_{0}(t)=t, P_{p}$ has real coefficients, and the degree of $P_{p}$ is $p$.

Thus $\operatorname{Res}\left(f_{p} ; Z_{s}\right)=\frac{e^{Z_{s}}}{Z_{s}^{(p+1) \alpha}} P_{p+1}\left(Z_{s}\right)$.

We write

$$
E_{\alpha, \alpha}^{(p)}(z)=\sum_{s \in \mathcal{S}} e^{Z_{s}} Z_{s}^{-(p+1) \alpha} P_{p+1}\left(Z_{s}\right)+\frac{p !}{2 \pi i} \int_{\gamma_{r}^{\prime}} e^{t} \frac{d t}{\left(t^{\alpha}-z\right)^{p+1}} .
$$

Next we estimate the second term on the right-hand in the above equality.

Observe that for any nonnegative integer $N$ we have

$$
\begin{aligned}
\frac{p !}{(1-u)^{p+1}} & =\frac{d^{p}}{d u^{p}}\left(\frac{1}{1-u}\right) \\
& =\sum_{k=p}^{N} \frac{k !}{(k-p) !} u^{k-p}+\sum_{l=0}^{p} p !\left(\begin{array}{c}
N+1 \\
p-l
\end{array}\right) u^{N+1-p+l} \frac{1}{(1-u)^{l+1}}
\end{aligned}
$$


This shows that

$$
\begin{aligned}
\frac{p !}{\left(t^{\alpha}-z\right)^{p+1}}= & (-1)^{p+1} \sum_{k=0}^{N-p} \frac{(k+p) !}{k !} \frac{t^{k \alpha}}{z^{k+p+1}} \\
& +\sum_{l=0}^{p} p !\left(\begin{array}{c}
N+1 \\
p-l
\end{array}\right)(-1)^{p+l} \frac{1}{z^{N+1}} \frac{t^{(N+1-p+l) \alpha}}{\left(t^{\alpha}-z\right)^{l+1}} .
\end{aligned}
$$

Integrating on $\gamma_{r}^{\prime}$ yields

$\frac{p !}{2 \pi i} \int_{\gamma_{r}^{\prime}} \frac{e^{t}}{\left(t^{\alpha}-z\right)^{p+1}} d t=(-1)^{p+1} \sum_{k=0}^{N-p} \frac{(k+p) !}{k !} \frac{1}{z^{k+p+1}} \frac{1}{2 \pi i} \int_{\gamma_{r}^{\prime}} e^{t} t^{k \alpha} d t+\mathcal{R}_{N}^{(p)}(z)$,

where

$$
\begin{aligned}
\mathcal{R}_{N}^{(p)}(z) & =\sum_{l=0}^{p} \mathcal{R}_{l, N}^{(p)}(z), \text { with } \mathcal{R}_{l, N}^{(p)}(z) \\
& =p !\left(\begin{array}{c}
N+1 \\
p-l
\end{array}\right)(-1)^{p+l} \frac{1}{z^{N+1}} \frac{1}{2 \pi i} \int_{\gamma_{r}^{\prime}} e^{t} \frac{t^{(N+1-p+l) \alpha}}{\left(t^{\alpha}-z\right)^{l+1}} d t
\end{aligned}
$$

Hence

$$
\frac{p !}{2 \pi i} \int_{\gamma_{r}^{\prime}} \frac{e^{t}}{\left(t^{\alpha}-z\right)^{p+1}} d t=(-1)^{p+1} \sum_{k=0}^{N-p} \frac{(k+p) !}{k !} \frac{1}{z^{k+p+1}} \frac{1}{\Gamma(-k \alpha)}+\mathcal{R}_{N}^{(p)}(z) .
$$

Since $\alpha$ is a positive integer, each term in the sum above is zero. First assume that for any integer $s,\left|\arg Z_{s}\right| \neq \pi$. When $r$ tends to 0 , if we choose $N$ such that $(N+1-p+l) \alpha>-1$, we can write, for $l \in\{0, \cdots, p\}$, $\mathcal{R}_{l, N}^{(p)}(z)=\mathcal{L}_{l, N}^{(p)}(z)+\mathcal{U}_{l, N}^{(p)}(z)$, where $\mathcal{L}_{l, N}^{(p)}(z)$ and $\mathcal{U}_{l, N}^{(p)}(z)$ are the integrals given by

$$
\begin{aligned}
\mathcal{L}_{l, N}^{(p)}(z)= & p !\left(\begin{array}{c}
N+1 \\
p-l
\end{array}\right)(-1)^{p+l} \frac{1}{z^{N+1}} \\
& \times \frac{1}{2 \pi i} \int_{+\infty}^{0} e^{-v} \frac{e^{-i \alpha \pi(N+1-p+l)} v^{(N+1-p+l) \alpha}}{\left(e^{-i \alpha \pi} v^{\alpha}-z\right)^{l+1}}(-d v), \\
\mathcal{U}_{l, N}^{(p)}(z)= & p !\left(\begin{array}{c}
N+1 \\
p-l
\end{array}\right)(-1)^{p+l} \frac{1}{z^{N+1}} \\
& \times \frac{1}{2 \pi i} \int_{0}^{+\infty} e^{-v} \frac{e^{i \alpha \pi(N+1-p+l)} v^{(N+1-p+l) \alpha}}{\left(e^{i \alpha \pi} v^{\alpha}-z\right)^{l+1}}(-d v)
\end{aligned}
$$


which can be rewritten

$\mathcal{L}_{l, N}^{(p)}(z)=p !\left(\begin{array}{c}N+1 \\ p-l\end{array}\right)(-1)^{p+l} \frac{1}{z^{N+1}} e^{-i \alpha \pi(N-p)} \frac{1}{2 \pi i} \int_{0}^{+\infty} \frac{e^{-v} v^{(N+1-p+l) \alpha}}{\left(v^{\alpha}-z e^{i \alpha \pi}\right)^{l+1}} d v$

and

$\mathcal{U}_{l, N}^{(p)}(z)=-p !\left(\begin{array}{c}N+1 \\ p-l\end{array}\right)(-1)^{p+l} \frac{1}{z^{N+1}} e^{i \alpha \pi(N-p)} \frac{1}{2 \pi i} \int_{0}^{+\infty} \frac{e^{-v} v^{(N+1-p+l) \alpha}}{\left(v^{\alpha}-z e^{-i \alpha \pi}\right)^{l+1}} d v$.

Since $\alpha$ is a positive integer, we see that $\mathcal{S}=\{0, \cdots, \alpha-1\}$ and $\mathcal{L}_{l, N}^{(p)}(z)=-\mathcal{U}_{l, N}^{(p)}(z)$ so that $\mathcal{R}_{l, N}^{(p)}(z)=0$ for $l \in\{0, \cdots, p\}$. We have the exact formula

$$
E_{\alpha, \alpha}^{(p)}(z)=\sum_{s \in \mathcal{S}} e^{Z_{s}} Z_{s}^{-(p+1) \alpha} P_{p+1}\left(Z_{s}\right)
$$

If there exists an integer $s_{0}$ such that $\left|\arg Z_{s_{0}}\right|=\pi$, the same identity holds by analytic continuation.

Lemma 3. Suppose $\alpha \in] 0,1[$ and $\epsilon$ is a positive number with $0<\sin \epsilon<$ $|\sin (\alpha \pi)|$. When $|\arg z| \leq \alpha \pi-\epsilon, \mathcal{S}=\{0\}$; when $\alpha \pi+\epsilon \leq|\arg z| \leq \pi, \mathcal{S}=$ $\varnothing$. In both cases, there exists a polynomial $P_{p+1}$ of degree $p+1$ defined in Lemma 2 and a non-negative integer $N$ such that for any $z \neq 0$,

$$
\begin{aligned}
E_{\alpha, \alpha}^{(p)}(z)= & \sum_{s \in \mathcal{S}} e^{Z_{s}} Z_{s}^{-\alpha(p+1)} P_{p+1}\left(Z_{s}\right) \\
& +\sum_{k=0}^{N-p}(-1)^{p+1} \frac{(k+p) !}{p !} \frac{1}{\Gamma(-k \alpha)} \frac{1}{z^{p+1+k}}+\mathcal{R}_{N}^{(p)}(z)
\end{aligned}
$$

and there exists a constant $C>0$, depending on $\alpha, p, N, \epsilon$ only such that for $|z| \geq 1\left|\mathcal{R}_{N}^{(p)}(z)\right| \leq \frac{C}{|z|^{N+2}}$.

Proof. Using the notations of Lemma 2 we obtain

$$
E_{\alpha, \alpha}^{(p)}(z)=\sum_{s \in \mathcal{S}} \operatorname{Res}\left(f_{p} ; Z_{s}\right)+\frac{1}{2 \pi i} \int_{\gamma_{r}^{\prime}} f_{p}(t) d t
$$

and

$$
\frac{p !}{2 \pi i} \int_{\gamma_{r}^{\prime}} \frac{e^{t}}{\left(t^{\alpha}-z\right)^{p+1}} d t=(-1)^{p+1} \sum_{k=0}^{N-p} \frac{(k+p) !}{k !} \frac{1}{z^{k+p+1}} \frac{1}{\Gamma(-k \alpha)}+\mathcal{R}_{N}^{(p)}(z),
$$


where

$$
\begin{aligned}
\mathcal{R}_{N}^{(p)}(z) & =\sum_{l=0}^{p} \mathcal{R}_{l, N}^{(p)}(z) \text { with } \mathcal{R}_{l, N}^{(p)}(z) \\
& =p !\left(\begin{array}{c}
N+1 \\
p-l
\end{array}\right)(-1)^{p+l} \frac{1}{z^{N+1}} \frac{1}{2 \pi i} \int_{\gamma_{r}^{\prime}} e^{t} \frac{t^{(N+1-p+l) \alpha}}{\left(t^{\alpha}-z\right)^{l+1}} d t .
\end{aligned}
$$

First assume that for any integer $s,\left|\arg Z_{s}\right| \neq \pi$. When $r$ tends to 0 , if we choose $N$ such that $(N+1-p+l) \alpha>-1$, we can write, for $l \in\{0, \cdots, p\}$, $\mathcal{R}_{l, N}^{(p)}(z)=\mathcal{L}_{l, N}^{(p)}(z)+\mathcal{U}_{l, N}^{(p)}(z)$, where $\mathcal{L}_{l, N}^{(p)}(z)$ and $\mathcal{U}_{l, N}^{(p)}(z)$ are the following integrals:

$$
\begin{aligned}
& \mathcal{L}_{l, N}^{(p)}(z)=p !\left(\begin{array}{c}
N+1 \\
p-l
\end{array}\right)(-1)^{p+l} \frac{1}{z^{N+1}} e^{-i \alpha \pi(N-p)} \frac{1}{2 \pi i} \int_{0}^{+\infty} \frac{e^{-v} v^{(N+1-p+l) \alpha}}{\left(v^{\alpha}-z e^{i \alpha \pi}\right)^{l+1}} d v \\
& \mathcal{U}_{l, N}^{(p)}(z)=-p !\left(\begin{array}{c}
N+1 \\
p-l
\end{array}\right)(-1)^{p+l} \frac{1}{z^{N+1}} e^{i \alpha \pi(N-p)} \frac{1}{2 \pi i} \int_{0}^{+\infty} \frac{e^{-v} v^{(N+1-p+l) \alpha}}{\left(v^{\alpha}-z e^{-i \alpha \pi}\right)^{l+1}} d v
\end{aligned}
$$

We distinguish the three cases corresponding to different values of $\arg z$.

First suppose $-\pi \leq \arg z \leq-\alpha \pi-\epsilon$. We determine the set $\mathcal{S}$. If $s$ is an integer put $\theta_{s}:=\frac{1}{\alpha}(\arg z+2 \pi s)$. We have $\frac{\pi}{\alpha}(-1+2 s) \leq \theta_{s} \leq-\pi-\frac{\epsilon}{\alpha}+\frac{2 \pi s}{\alpha}$. If $s \leq 0$, then $\theta_{s} \leq-\pi-\frac{\epsilon}{\alpha}<-\pi$, and hence $s \notin \mathcal{S}$. Otherwise, $s \geq 1$ and thus $\theta_{s} \geq \frac{\pi}{\alpha}$; it follows that $s \notin \mathcal{S}$. This shows $\mathcal{S}=\varnothing$. Since $-\pi+\alpha \pi \leq \arg \left(z e^{i \alpha \pi}\right) \leq-\epsilon$, we see that $\left|z e^{i \alpha \pi}-v^{\alpha}\right| \geq \sin \epsilon|z|$ for any real positive number $v$.

In the same way, for the case $-\pi-\alpha \pi \leq \arg \left(z e^{-i \alpha \pi}\right) \leq-2 \alpha \pi-\epsilon$, we obtain that for any $v$ positive number

$$
\left|z e^{-i \alpha \pi}-v^{\alpha}\right| \geq \sin (\alpha \pi)|z|>\sin \epsilon|z| .
$$

Similar arguments show that for the remaining cases $-\alpha \pi+\epsilon \leq \arg z \leq$ $\alpha \pi-\epsilon$, we have $\mathcal{S}=\{0\}$ and $\alpha \pi+\epsilon \leq \arg z \leq \pi$, we have $\mathcal{S}=\varnothing$. Moreover, (3.1) holds in all cases.

Hence for $l \in\{0, \cdots, p\}$ both $\mathcal{L}_{l, N}^{(p)}(z)$ and $\mathcal{U}_{l, N}^{(p)}(z)$ satisfy the same inequality

$$
\begin{aligned}
& \left|\mathcal{L}_{l, N}^{(p)}(z)\right| \leq \frac{p !\left(\begin{array}{c}
N+1 \\
p-l
\end{array}\right)}{(\sin \epsilon)^{l+1}} \frac{\Gamma((N+1-p+l) \alpha+1)}{2 \pi} \frac{1}{|z|^{N+2+l}}, \\
& \left|\mathcal{U}_{l, N}^{(p)}(z)\right| \leq \frac{p !\left(\begin{array}{c}
N+1 \\
p-l
\end{array}\right)}{(\sin \epsilon)^{l+1}} \frac{\Gamma((N+1-p+l) \alpha+1)}{2 \pi} \frac{1}{|z|^{N+2+l}},
\end{aligned}
$$


and

$$
\left|\mathcal{R}_{l, N}^{(p)}(z)\right| \leq \frac{p !\left(\begin{array}{c}
N+1 \\
p-l
\end{array}\right)}{(\sin \epsilon)^{l+1}} \frac{\Gamma((N+1-p+l) \alpha+1)}{\pi} \frac{1}{|z|^{N+2+l}} .
$$

The required result follows by taking the sum over $l \in\{0, \cdots, p\}$. Now assume that there exists an integer $s_{0}$ such that $\left|\arg Z_{s_{0}}\right|=\pi$. We choose $r$ sufficiently small so that the poles $Z_{s}$ with $s \in \mathcal{S}$ are to the right of $\gamma_{r}^{\prime}$. Recall that $E_{\alpha, \alpha}^{(p)}(z)=\sum_{s \in \mathcal{S}} e^{Z_{s}} Z_{s}^{-(p+1) \alpha} P_{p+1}\left(Z_{s}\right)+\frac{p !}{2 \pi i} \int_{\gamma_{r}^{\prime}} e^{t} \frac{d t}{\left(t^{\alpha}-z\right)^{p+1}}$.

We deform $\gamma_{r}^{\prime}$ in another Hankel contour $\gamma_{r, a^{\prime}}^{\prime}$ surrounding the singularity $Z_{s_{0}}$ from above and below by circular parts of radius $a^{\prime}$ such that the poles $Z_{s}$ with $s \in \mathcal{S}$ are to the right of $\gamma_{r, a^{\prime}}^{\prime}$. We have

$$
\frac{p !}{2 \pi i} \int_{\gamma_{r}^{\prime}} e^{t} \frac{d t}{\left(t^{\alpha}-z\right)^{p+1}}=\frac{p !}{2 \pi i} \int_{\gamma_{r, a^{\prime}}^{\prime}} e^{t} \frac{d t}{\left(t^{\alpha}-z\right)^{p+1}} .
$$

Following the same method as above we get

$$
E_{\alpha, \alpha}^{(p)}(z)=\sum_{s \in \mathcal{S}} e^{Z_{s}} Z_{s}^{-(p+1) \alpha} P_{p+1}\left(Z_{s}\right)+\frac{p !}{2 \pi i} \int_{\gamma_{r, \alpha^{\prime}}^{\prime}} e^{t} \frac{d t}{\left(t^{\alpha}-z\right)^{p+1}}
$$

and

$\frac{p !}{2 \pi i} \int_{\gamma_{r, a^{\prime}}^{\prime}} \frac{e^{t}}{\left(t^{\alpha}-z\right)^{p+1}} d t=(-1)^{p+1} \sum_{k=0}^{N-p} \frac{(k+p) !}{k !} \frac{1}{z^{k+p+1}} \frac{1}{\Gamma(-k \alpha)}+\mathcal{R}_{N}^{(p)}(z)$,

where

$$
\begin{aligned}
\mathcal{R}_{N}^{(p)}(z) & =\sum_{l=0}^{p} \mathcal{R}_{l, N}^{(p)}(z) \text { with } \mathcal{R}_{l, N}^{(p)}(z) \\
& =p !\left(\begin{array}{c}
N+1 \\
p-l
\end{array}\right)(-1)^{p+l} \frac{1}{z^{N+1}} \frac{1}{2 \pi i} \int_{\gamma_{r, a^{\prime}}^{\prime}} e^{t} \frac{t^{(N+1-p+l) \alpha}}{\left(t^{\alpha}-z\right)^{l+1}} d t
\end{aligned}
$$

If $r$ tends to $0, \gamma_{r, a^{\prime}}^{\prime}$ is deformed to $\gamma_{0, a^{\prime}}$ which consists of the following four disconnected lines segments, the first one starts below the negative real axis at $-\infty$ to $Z_{s_{0}}-a^{\prime}$, the second one joins $Z_{s_{0}}+a^{\prime}$ to 0 , the third joins 0 to $Z_{s_{0}}+a^{\prime}$ above the negative real axis and the fourth returning from $Z_{s_{0}}-a^{\prime}$ to $-\infty$. Then we see that 


$$
\begin{aligned}
\lim _{r \rightarrow 0} \frac{1}{2 \pi i} \int_{\gamma_{r, a^{\prime}}^{\prime}} e^{t} \frac{t^{(N+1-p+l) \alpha}}{\left(t^{\alpha}-z\right)^{l+1}} d t= & \operatorname{Res}\left(e^{t} \frac{t^{(N+1-p+l) \alpha}}{\left(t^{\alpha}-z\right)^{l+1}} ; t=Z_{s_{0}}\right) \\
& +\frac{1}{2 \pi i} \int_{\gamma_{0, a^{\prime}}^{\prime}} e^{t} \frac{t^{(N+1-p+l) \alpha}}{\left(t^{\alpha}-z\right)^{l+1}} d t
\end{aligned}
$$

Let $\mathcal{C}_{s_{0}}$ be a circle centered at $Z_{s_{0}}$ of radius $\eta$. Then we have

$$
\operatorname{Res}\left(e^{t} \frac{t^{(N+1-p+l) \alpha}}{\left(t^{\alpha}-z\right)^{l+1}} ; t=Z_{s_{0}}\right)=\frac{1}{2 \pi i} \int_{\mathcal{C}_{s_{0}}} e^{t} \frac{t^{(N+1-p+l) \alpha}}{\left(t^{\alpha}-z\right)^{l+1}} d t .
$$

Let be $\mathcal{C}_{s_{0}}^{\prime}$ the image of $\mathcal{C}_{s_{0}}$ by the function power $\alpha$, where $\eta$ is chosen small enough so that $\mathcal{C}_{s}$ and $\mathcal{C}_{s_{0}}^{\prime}$ are included in $\mathbb{C} \backslash \mathbb{R}_{+}$and $u=t^{\alpha}=$ $|t|^{\alpha} \exp \left[i \alpha\left(\arg t+2 \pi s_{0}\right)\right]$, where $0<\arg t<2 \pi$. In a neighbourhood of $\mathcal{C}_{s_{0}}^{\prime}$, we define $u^{\frac{1}{\alpha}}=|u|^{\frac{1}{\alpha}} \exp \left[\frac{i}{\alpha}(\arg u+2 \pi s)\right]$, where $0<\arg u<2 \pi$. The change of variables $u=t^{\alpha}$ shows that

$$
\begin{aligned}
\operatorname{Res}\left(e^{t} \frac{t^{(N+1-p+l) \alpha}}{\left(t^{\alpha}-z\right)^{l+1}} ; t=Z_{s_{0}}\right) & =\operatorname{Res}\left(\frac{1}{\alpha} e^{u^{\frac{1}{\alpha}}} \frac{u^{N-p+l+\frac{1}{\alpha}}}{(u-z)^{l+1}} ; u=z\right) \\
& =\frac{1}{l !} \frac{d^{l}}{d u^{l}}\left(\frac{1}{\alpha} e^{u^{\frac{1}{\alpha}}} u^{N-p+l+\frac{1}{\alpha}}\right)_{u=z} \\
& =e^{Z_{s_{0}}} Q_{N-p, l}\left(Z_{s_{0}}^{\alpha}, Z_{s_{0}}\right),
\end{aligned}
$$

for some polynomial $Q_{N-p, l}$. Thus

$$
\begin{aligned}
\mathcal{R}_{l, N}^{(p)}(z)=p ! & \left(\begin{array}{c}
N+1 \\
p-l
\end{array}\right)(-1)^{p+l} \frac{1}{z^{N+1}}\left[e^{Z_{s_{0}}} Q_{N-p, l}\left(Z_{s_{0}}^{\alpha}, Z_{s_{0}}\right)\right. \\
& \left.+\frac{1}{2 \pi i} \int_{\gamma_{0, a^{\prime}}^{\prime}} e^{t} \frac{t^{(N+1-p+l) \alpha}}{\left(t^{\alpha}-z\right)^{l+1}} d t\right]
\end{aligned}
$$

Since $Z_{s_{0}}$ is a negative real number and the integral $\frac{1}{2 \pi i} \int_{\gamma_{0, a^{\prime}}^{\prime}} e^{t} \frac{t^{(N+1-p+l) \alpha}}{\left(t^{\alpha}-z\right)^{l+1}} d t$ can be handled in the same way as the integral $\frac{1}{2 \pi i} \int_{\gamma_{r}^{\prime}} e^{t} \frac{t^{(N+1-p+l) \alpha}}{\left(t^{\alpha}-z\right)^{l+1}} d t$ previously treated, we see that for any $l \in\{0, \ldots, p\}, \mathcal{R}_{l, N}^{(p)}(z)$ satisfies a similar estimate. Hence for the ranges $|\arg z| \leq \alpha \pi-\epsilon$ and $\alpha \pi+\epsilon \leq$ $|\arg z| \leq \pi$, there exists a constant $C$ depending on $\alpha, p, N$ only such that when $|z| \geq 1,\left|\mathcal{R}_{N}^{(p)}(z)\right| \leq \frac{C}{|z|^{N+2}}$. 
Lemma 4. Suppose $\alpha \in] 2 L-1,2 L[$, where $L$ is a positive integer, and $\epsilon$ is a positive number with $0<\sin \epsilon<|\sin (\alpha \pi)|$.

(i) If $-\pi \leq \arg z \leq-(2 L-\alpha) \pi-\epsilon$, then $\mathcal{S}=\{-(L-1), \cdots, 0, \cdots, L\}$.

(ii) If $|\arg z| \leq(2 L-\alpha) \pi-\epsilon$, then $\mathcal{S}=\{-(L-1), \cdots, 0, \cdots, L-1\}$

(iii) If $(2 L-\alpha) \pi+\epsilon \leq \arg z \leq \pi$, then $\mathcal{S}=\{-L, \cdots, 0, \cdots, L-1\}$

In those cases, there exists a polynomial $P_{p+1}$ of degree $p+1$ defined in Lemma 2 and a non-negative $N$ such that for any $z \neq 0$,

$$
\begin{aligned}
E_{\alpha, \alpha}^{(p)}(z)= & \sum_{s \in \mathcal{S}} e^{Z_{s}} Z_{s}^{-\alpha(p+1)} P_{p+1}\left(Z_{s}\right) \\
& +\sum_{k=0}^{N-p}(-1)^{p+1} \frac{(k+p) !}{p !} \frac{1}{\Gamma(-k \alpha)} \frac{1}{z^{p+1+k}}+\mathcal{R}_{N}^{(p)}(z)
\end{aligned}
$$

and there exists a constant $C>0$, depending on $\alpha, p, N, \epsilon$ only such that

$$
\left|\mathcal{R}_{N}^{(p)}(z)\right| \leq \frac{C}{|z|^{N+2}}, \text { for }|z| \geq 1
$$

Proof. Arguing as in Lemma 2 we have

$$
E_{\alpha, \alpha}^{(p)}(z)=\sum_{s \in \mathcal{S}} \operatorname{Res}\left(f_{p} ; Z_{s}\right)+\frac{1}{2 \pi i} \int_{\gamma_{r}^{\prime}} f_{p}(t) d t
$$

and

$$
\frac{p !}{2 \pi i} \int_{\gamma_{r}^{\prime}} \frac{e^{t}}{\left(t^{\alpha}-z\right)^{p+1}} d t=(-1)^{p+1} \sum_{k=0}^{N-p} \frac{(k+p) !}{k !} \frac{1}{z^{k+p+1}} \frac{1}{\Gamma(-k \alpha)}+\mathcal{R}_{N}^{(p)}(z),
$$

where $\mathcal{R}_{N}^{(p)}(z)=\sum_{l=0}^{p} \mathcal{R}_{l, N}^{(p)}(z)$ with

$$
\mathcal{R}_{l, N}^{(p)}(z)=p !\left(\begin{array}{c}
N+1 \\
p-l
\end{array}\right)(-1)^{p+l} \frac{1}{z^{N+1}} \frac{1}{2 \pi i} \int_{\gamma_{r}^{\prime}} e^{t} \frac{t^{(N+1-p+l) \alpha}}{\left(t^{\alpha}-z\right)^{l+1}} d t .
$$

First assume that for any integer $s,\left|\arg Z_{s}\right| \neq \pi$. Choose $N$ such that $(N+1-p+l) \alpha>-1$. We can write, for $l \in\{0, \cdots, p\}, \mathcal{R}_{l, N}^{(p)}(z)=$ $\mathcal{L}_{l, N}^{(p)}(z)+\mathcal{U}_{l, N}^{(p)}(z)$, where

$$
\mathcal{L}_{l, N}^{(p)}(z)=p !\left(\begin{array}{c}
N+1 \\
p-l
\end{array}\right)(-1)^{p+l} \frac{1}{z^{N+1}} e^{-i \alpha \pi(N-p)} \frac{1}{2 \pi i} \int_{0}^{+\infty} \frac{e^{-v} v^{(N+1-p+l) \alpha}}{\left(v^{\alpha}-z e^{i \alpha \pi}\right)^{l+1}} d v
$$


$\mathcal{U}_{l, N}^{(p)}(z)=-p !\left(\begin{array}{c}N+1 \\ p-l\end{array}\right)(-1)^{p+l} \frac{1}{z^{N+1}} e^{i \alpha \pi(N-p)} \frac{1}{2 \pi i} \int_{0}^{+\infty} \frac{e^{-v} v^{(N+1-p+l) \alpha}}{\left(v^{\alpha}-z e^{-i \alpha \pi}\right)^{l+1}} d v$

For the different ranges of $\arg z$, namely $-\pi \leq \arg z \leq-(2 L-\alpha) \pi-\epsilon$, $-(2 L-\alpha) \pi+\epsilon \leq \arg z \leq+(2 L-\alpha) \pi-\epsilon$, and $(2 L-\alpha) \pi+\epsilon \leq \arg z \leq \pi$, the set $\mathcal{S}$ is easy to determine. In each case, for a positive number $v$, the inequality (3.1) holds. Therefore we get, for $l \in\{0, \cdots, p\}$

$$
\begin{gathered}
\left|\mathcal{L}_{l, N}^{(p)}(z)\right| \leq \frac{p !\left(\begin{array}{c}
N+1 \\
p-l
\end{array}\right)}{(\sin \epsilon)^{l+1}} \frac{\Gamma((N+1-p+l) \alpha+1)}{2 \pi} \frac{1}{|z|^{N+2+l}} \\
\left|\mathcal{L}_{l, N}^{(p)}(z)\right| \leq \frac{p !\left(\begin{array}{c}
N+1 \\
p-l
\end{array}\right)}{(\sin \epsilon)^{l+1}} \frac{\Gamma((N+1-p+l) \alpha+1)}{2 \pi} \frac{1}{|z|^{N+2+l}},
\end{gathered}
$$

and

$$
\left|\mathcal{R}_{l, N}^{(p)}(z)\right| \leq \frac{p !\left(\begin{array}{c}
N+1 \\
p-l
\end{array}\right)}{(\sin \epsilon)^{l+1}} \frac{\Gamma((N+1-p+l) \alpha+1)}{\pi} \frac{1}{|z|^{N+2+l}} .
$$

Taking the sum over $l \in\{0, \cdots, p\}$ we get the required result.

Now we consider the results in the case when $z$ is a negative real number. Notice that if $\arg z=-\pi$, then $\arg Z_{s}=\frac{\pi}{\alpha}(-1+2 s)$ with $s \in\{-(L-1), \ldots, 0, \ldots, L\}$ and if $\arg z=\pi$, then $\arg Z_{s}=\frac{\pi}{\alpha}(1+2 s)$ with $s \in\{-L, \ldots, 0, \ldots, L-1\}$. It follows that $\left\{Z_{s}, s \in\{-(L-\right.$ $1), \ldots, 0, \ldots, L\}\}=\left\{Z_{s}, s \in\{-L, \ldots, 0, \ldots, L-1\}\right\}$. Therefore when $z$ is a negative real number, the formulas obtained in the first and the third subcases coincide.

Finally if there exists an integer $s_{0}$ such that $\left|\arg Z_{s_{0}}\right|=\pi$, the proof is similar to that of Lemma 3.

Lemma 5. Suppose $\alpha \in] 2 L, 2 L+1[$, where $L$ is a positive integer, and $\epsilon$ is a positive number with $0<\sin \epsilon<|\sin (\alpha \pi)|$.

(i) If $-\pi \leq \arg z \leq-(\alpha-2 L) \pi-\epsilon$, then $\mathcal{S}=\{-(L-1), \cdots, 0, \cdots, L\}$.

(ii) If $|\arg z| \leq(\alpha-2 L) \pi-\epsilon$, then $\mathcal{S}=\{-(L-1), \cdots, 0, \cdots,(L-1)\}$.

(iii) If $(\alpha-2 L) \pi+\epsilon \leq \arg z \leq \pi$, then $\mathcal{S}=\{-L, \cdots, 0, \cdots, L-1\}$.

In those cases, there exists a polynomial $P_{p+1}$ of degree $p+1$ defined in Lemma 2 and a non-negative integer $N$ such that for any $z \neq 0$,

$$
\begin{aligned}
E_{\alpha, \alpha}^{(p)}(z)= & \sum_{s \in \mathcal{S}} e^{Z_{s}} Z_{s}^{-\alpha(p+1)} P_{p+1}\left(Z_{s}\right) \\
& +\sum_{k=0}^{N-p}(-1)^{p+1} \frac{(k+p) !}{p !} \frac{1}{\Gamma(-k \alpha)} \frac{1}{z^{p+1+k}}+\mathcal{R}_{N}^{(p)}(z)
\end{aligned}
$$


and there exists a constant $C>0$, depending on $\alpha, p, N, \epsilon$ only such that

$$
\left|\mathcal{R}_{N}^{(p)}(z)\right| \leq \frac{C}{|z|^{N+2}} \text { for }|z| \geq 1 .
$$

Proof. Proceeding as in Lemma 2 we get again

$$
E_{\alpha, \alpha}^{(p)}(z)=\sum_{s \in \mathcal{S}} \operatorname{Res}\left(f_{p} ; Z_{s}\right)+\frac{1}{2 \pi i} \int_{\gamma_{r}^{\prime}} f_{p}(t) d t
$$

and

$$
\frac{p !}{2 \pi i} \int_{\gamma_{r}^{\prime}} \frac{e^{t}}{\left(t^{\alpha}-z\right)^{p+1}} d t=(-1)^{p+1} \sum_{k=0}^{N-p} \frac{(k+p) !}{k !} \frac{1}{z^{k+p+1}} \frac{1}{\Gamma(-k \alpha)}+\mathcal{R}_{N}^{(p)}(z),
$$

where $\mathcal{R}_{N}^{(p)}(z)=\sum_{l=0}^{p} \mathcal{R}_{l, N}^{(p)}(z)$ with

$$
\mathcal{R}_{l, N}^{(p)}(z)=p !\left(\begin{array}{c}
N+1 \\
p-l
\end{array}\right)(-1)^{p+l} \frac{1}{z^{N+1}} \frac{1}{2 \pi i} \int_{\gamma_{r}^{\prime}} e^{t} \frac{t^{(N+1-p+l) \alpha}}{\left(t^{\alpha}-z\right)^{l+1}} d t
$$

First we consider the case $\left|\arg Z_{s}\right| \neq \pi$ for all integers $s$. Choosing $N$ such that $(N+1-p+l) \alpha>-1$, we can write, for $l \in\{0, \cdots, p\}$, $\mathcal{R}_{l, N}^{(p)}(z)=\mathcal{L}_{l, N}^{(p)}(z)+\mathcal{U}_{l, N}^{(p)}(z)$, where

$\mathcal{L}_{l, N}^{(p)}(z)=p !\left(\begin{array}{c}N+1 \\ p-l\end{array}\right)(-1)^{p+l} \frac{1}{z^{N+1}} e^{-i \alpha \pi(N-p)} \frac{1}{2 \pi i} \int_{0}^{+\infty} \frac{e^{-v} v^{(N+1-p+l) \alpha}}{\left(v^{\alpha}-z e^{i \alpha \pi}\right)^{l+1}} d v$

$\mathcal{U}_{l, N}^{(p)}(z)=-p !\left(\begin{array}{c}N+1 \\ p-l\end{array}\right)(-1)^{p+l} \frac{1}{z^{N+1}} e^{i \alpha \pi(N-p)} \frac{1}{2 \pi i} \int_{0}^{+\infty} \frac{e^{-v} v^{(N+1-p+l) \alpha}}{\left(v^{\alpha}-z e^{-i \alpha \pi}\right)^{l+1}} d v$

The set $\mathcal{S}$ can then be determined as in Lemmas 3 and 4 and the estimate for $\left|\mathcal{R}_{N}^{(p)}(z)\right|$ is similar. If $z$ is a negative real number, we observe that

$$
\left\{Z_{s}, s \in\{-(L-1), \ldots, 0, \ldots, L\}\right\}=\left\{Z_{s}, s \in\{-L, \ldots, 0, \ldots, L-1\}\right\} .
$$

Thus the formulas obtained for $-\pi \leq \arg z \leq-(\alpha-2 L) \pi-\epsilon$ and $(\alpha-2 L) \pi+\epsilon \leq \arg z \leq \pi$ coincide.

If $\left|\arg Z_{s_{0}}\right|=\pi$ for some integer $s_{0}$, then the proof is the same as in Lemma 3 .

Recall that

$$
\rho_{m}(a, b)=\frac{N(a, b)}{D(a, b)},
$$


where $N(a, b)=F\left(|a|^{2}\right) F\left(|b|^{2}\right)-|F(\langle b, a\rangle)|^{2}$ and $D(a, b)=F\left(|a|^{2}\right) F\left(|b|^{2}\right)$.

We first estimate the numerator by using a Taylor expansion near $a$. To do so we need to study $F$ and its higher order derivatives near the positive real axis.

Lemma 6. Let $p$ be a non-negative integer. There exists a constant $\epsilon>0$ such that, for any complex $z$ with $|\arg z| \leq \epsilon$, we have

$$
F^{(p)}(z)=e^{Z_{0}} Z_{0}^{-(n+p) \alpha} P_{n+p}\left(Z_{0}\right)+F_{p}(z),
$$

where $Z_{s}=|z|^{\frac{1}{\alpha}} \exp \left[\frac{i}{\alpha}(\arg z+2 \pi s)\right]$, for $s$ integer, the polynomials $\left(P_{p}\right)_{p \in \mathbb{N}}$ are defined by the relation

$$
\frac{d^{p}}{d u^{p}}\left[e^{u^{\frac{1}{\alpha}}}\right]=\frac{e^{u^{\frac{1}{\alpha}}}}{u^{p}} P_{p}\left(u^{\frac{1}{\alpha}}\right)
$$

and $F_{p}(z)=\left\{\begin{array}{ll}O\left(\frac{1}{|z|}\right)^{p+1} & \text { if } \alpha \in] 0,1], \\ O\left(|z|^{q} e^{Z_{ \pm 1}}\right) & \text { for any integer } q \text { if } \alpha>1\end{array}\right.$.

Proof. Follows from Lemmas 2, 3, 4, 5 and the fact that when $\arg z$ tends to 0 , with $s$ in $\mathcal{S}$ such that $|s| \geq 2$ and $q, q^{\prime}, q^{\prime \prime}$ are integers, $e^{Z_{s}}|z|^{q^{\prime \prime}}$ is negligible in comparison of $e^{Z_{ \pm 1}}|z|^{q^{\prime}}$ and $e^{Z_{ \pm 1}}|z|^{q^{\prime}}$ is negligible in comparison of $e^{Z_{0}}|z|^{q}$.

We will also need some properties of the polynomials $\left(P_{p}\right)_{p \in \mathbb{N}}$.

Lemma 7. The sequence $\left(P_{p}\right)_{p \in \mathbb{N}}$ defined by (3.2) satisfies the recurrence relation:

$$
P_{p+1}(t)=\left(\frac{t}{\alpha}-p\right) P_{p}(t)+\frac{t}{\alpha} P_{p}^{\prime}(t) .
$$

In particular each $P_{p}$ has real coefficients, and is of degree $p$. In addition, the polynomial $P_{p}(t)-\left[\frac{1}{\alpha^{p}} t^{p}+\frac{p(p-1)}{2 \alpha^{p-1}}\left(\frac{1}{\alpha}-1\right) t^{p-1}\right]$ is of degree at most $p-2$ and satisfies

$$
\left(P_{p} P_{p+2}-P_{p+1}^{2}\right)(t) \sim \frac{1}{\alpha^{2 p+1}}\left(\frac{1}{\alpha}-1\right) t^{2 p+1}, t \rightarrow+\infty
$$

Proof. The relation between the polynomials $\left(P_{p}\right)$ and the fact that $P_{p}$ has real coefficients and degree $p$ have been shown in the proof of the Lemma 2. We observe that the term of highest degree term of $P_{p}$ is $\frac{1}{\alpha^{p}} t^{p}$. 
Then $P_{p}$ can be written in the form

$$
P_{p}(t)=\frac{1}{\alpha^{p}} t^{p}+b_{p} t^{p-1}+\widetilde{P}_{p}(t)
$$

where $\widetilde{P}_{p}(t)$ has a degree at most $p-2$. We need to compute $b_{p}$. By differentiation we have $P_{p}^{\prime}(t)=\frac{p}{\alpha^{p}} t^{p-1}+(p-1) b_{p} t^{p-2}+\widetilde{P}_{p}^{\prime}(t)$, and therefore

$$
P_{p+1}(t)=\frac{1}{\alpha^{p+1}} t^{p+1}+t^{p}\left[\frac{b_{p}}{\alpha}+\frac{p}{\alpha^{p+1}}-\frac{p}{\alpha^{p}}\right]+\widetilde{P}_{p+1}(t) .
$$

It follows from this that $b_{p+1}=\frac{b_{p}}{\alpha}+\frac{p}{\alpha^{p}}\left(\frac{1}{\alpha}-1\right)$.

Since $P_{1}(t)=\frac{1}{\alpha} t$, we see that $b_{1}=0$; an induction process gives

$$
b_{p}=\frac{p(p-1)}{2 \alpha^{p-1}}\left(\frac{1}{\alpha}-1\right) .
$$

Using the identities

$$
P_{q}(t)=\frac{1}{\alpha^{q}} t^{q}+\frac{q(q-1)}{2 \alpha^{q-1}}\left(\frac{1}{\alpha}-1\right) t^{q-1}+\widetilde{P}_{q}(t), \text { for } q=p, p+1, p+2,
$$

we find that the term of highest degree in $P_{p+2} P_{p}-P_{p+1}^{2}$ is $\frac{1}{\alpha^{2 p+1}}\left(\frac{1}{\alpha}-1\right) t^{2 p+1}$.

\section{Preparatory estimates}

To study $\rho_{m}(a, a+u)$, we first focus on the numerator $N(a, a+u)$.

Lemma 8. There are positive constants $A$ and $C$ such that for any $a$, $u$ in $\mathbb{C}^{n}$ with $|a|>A$ and $|u|<1$, we have

$$
N(a, a+u)=\left(F F^{\prime}\right)\left(|a|^{2}\right)|u|^{2}+\left(F F^{\prime \prime}-F^{\prime 2}\right)\left(|a|^{2}\right)|\langle u, a\rangle|^{2}+R(a, u),
$$

where

$$
|R(a, u)| \leq C e^{2|a|^{\frac{2}{\alpha}}}|a|^{\frac{2}{\alpha}(1-\alpha) 2 n}\left[|a|^{\frac{4}{\alpha}-3}+|a|^{\frac{6}{\alpha}-3}+|a|^{\frac{8}{\alpha}-4}+|a|^{\frac{10}{\alpha}-5}+|a|^{\frac{12}{\alpha}-6}\right]|u|^{3} .
$$

Proof. The Taylor expansion formula gives

$$
\begin{aligned}
F\left(|a+u|^{2}\right)= & F\left(|a|^{2}\right)+F^{\prime}\left(|a|^{2}\right)\left(\langle u, a\rangle+\langle a, u\rangle+|u|^{2}\right) \\
& +\frac{1}{2} F^{\prime \prime}\left(|a|^{2}\right)(\langle u, a\rangle+\langle a, u\rangle)^{2}+R_{1}(a, u),
\end{aligned}
$$


and

$$
F(\langle a, a+u\rangle)=F\left(|a|^{2}\right)+F^{\prime}\left(|a|^{2}\right)\langle a, u\rangle+\frac{1}{2} F^{\prime \prime}\left(|a|^{2}\right)(\langle a, u\rangle)^{2}+R_{2}(a, u),
$$

where

$$
\begin{aligned}
& R_{1}(a, u)=\frac{1}{2} F^{\prime \prime}\left(|a|^{2}\right)\left(|u|^{4}+2|u|^{2}(\langle u, a\rangle+\langle a, u\rangle)\right) \\
+ & \frac{1}{2} \int_{0}^{1}(1-t)^{2} F^{(3)}\left(|a|^{2}+t\left(|u|^{2}+\langle u, a\rangle+\langle a, u\rangle\right)\left(\langle u, a\rangle+\langle a, u\rangle+|u|^{2}\right)^{3} d t .\right.
\end{aligned}
$$

and

$$
R_{2}(a, u)=\frac{1}{2} \int_{0}^{1}(1-t)^{2} F^{(3)}\left(|a|^{2}+t\langle a, u\rangle\right)\langle a, u\rangle^{3} d t
$$

We write

$$
\begin{aligned}
& |F(\langle a, a+u\rangle)|^{2}=F^{2}\left(|a|^{2}\right)+\left(F F^{\prime}\right)\left(|a|^{2}\right)(\langle a, u\rangle+\langle u, a\rangle) \\
& \quad+|\langle a, u\rangle|^{2} F^{\prime 2}\left(|a|^{2}\right)+\frac{1}{2}\left(\langle a, u\rangle^{2}+\langle u, a\rangle^{2}\right)\left(F^{\prime} F^{\prime \prime}\right)\left(|a|^{2}\right)+R_{3}(a, u),
\end{aligned}
$$

and let $R(a, u)=F\left(|a|^{2}\right) R_{1}(a, u)-R_{3}(a, u)$. We get

$$
\left.N(a, a+u)=\left(F F^{\prime}\right)\left(|a|^{2}\right)|u|^{2}+\left(F F^{\prime \prime}-F^{\prime 2}\right)\left(|a|^{2}\right) \mid\langle a, u\rangle\right)\left.\right|^{2}+R(a, u) .
$$

We shall estimate the remainder $R(a, u)$. By Lemma 6 , there are positive constants $A_{1}, D_{1}, \epsilon$ such that for any $p=0, \ldots, 3$ and for any $z \in \mathbb{C}$ with $|\arg z|<\epsilon$ and $|z|>A_{1}$, we have

$$
\left|F^{(p)}(z)\right| \leq D_{1} e^{Z_{0}} Z_{0}^{(n+p)(1-\alpha)}, \text { where } Z_{0}=|z|^{\frac{1}{\alpha}} \exp \left[\frac{i}{\alpha} \arg z\right] .
$$

Notice that

$$
\left\{\begin{aligned}
\arg \left[|a|^{2}+t\left(|u|^{2}+2 \Re(\langle u, a\rangle)\right)\right] & =\arg \left[1+\frac{t}{|a|^{2}}\left(|u|^{2}+2 \Re(\langle u, a\rangle)\right)\right] \\
\arg \left[|a|^{2}+t\langle a, u\rangle\right] & =\arg \left[1+\frac{t}{|a|^{2}}\langle a, u\rangle\right] .
\end{aligned}\right.
$$

Thus there exists a positive constant $A_{2}$ such that for any $p=0, \ldots, 3$ and for any $a, u$ in $\mathbb{C}^{n}$ with $|a|>A_{2}$ and $|u|<1$, we have $\left|\arg \left[|a|^{2}+t\left(|u|^{2}+2 \Re(\langle u, a\rangle)\right)\right]\right|<\epsilon$, and $\left|\arg \left[|a|^{2}+t\langle a, u\rangle\right]\right|<\epsilon$. 
Therefore

$$
\left\{\begin{array}{l}
\left|F^{(p)}\left(|a|^{2}\right)\right| \leq D_{1} e^{|a|^{\frac{2}{\alpha}}}|a|^{\frac{2}{\alpha}(n+p)(1-\alpha)}, \\
\mid\left(F ^ { ( 3 ) } \left(|a|^{2}+\left.t\left(|u|^{2}+2 \Re(\langle u, a\rangle)\right)\left|\leq D_{1} e^{|a|^{\frac{2}{\alpha}}}\right| a\right|^{\frac{2}{\alpha}(n+3)(1-\alpha)} \text { for any } t \in[0,1],\right.\right. \\
\left|F^{(3)}\left(|a|^{2}+t\langle a, u\rangle\right)\right| \leq D_{1} e^{|a|^{\frac{2}{\alpha}}}|a|^{\frac{2}{\alpha}(n+3)(1-\alpha)}
\end{array}\right.
$$

It follows that

$$
\begin{gathered}
|R(a, u)| \leq D_{1}^{2} e^{2|a|^{\frac{2}{\alpha}}}|a|^{\frac{2}{\alpha}(1-\alpha) 2 n}\left[(4|a|+|u|)|a|^{\frac{4}{\alpha}(1-\alpha)}+(2|a|+|u|)^{3}|a|^{\frac{6}{\alpha}(1-\alpha)}\right. \\
+|a|^{3}|a|^{\frac{6}{\alpha}(1-\alpha)}+2|a|^{4}|a|^{\frac{8}{\alpha}(1-\alpha)}|u|+2|a|^{3}|a|^{\frac{6}{\alpha}(1-\alpha)} \\
\left.+|a|^{5}|a|^{\frac{10}{\alpha}(1-\alpha)}|u|^{2}+|a|^{6}|a|^{\frac{12}{\alpha}(1-\alpha)}|u|^{3}\right]|u|^{3} .
\end{gathered}
$$

Hence there exist positive constants $A_{3}, C$ such that for any $a, u$ in $\mathbb{C}^{n}$ with $|a|>A_{3}$ and $|u|<1$, we have

$|R(a, u)| \leq C e^{2|a|^{\frac{2}{\alpha}}}|a|^{\frac{2}{\alpha}(1-\alpha)(2 n)}\left[|a|^{\frac{4}{\alpha}-3}+|a|^{\frac{6}{\alpha}-3}+|a|^{\frac{8}{\alpha}-4}+|a|^{\frac{10}{\alpha}-5}+|a|^{\frac{12}{\alpha}-6}\right]|u|^{3}$,

and the proof is complete.

We next need to study the denominator $D(a, a+u)$ of $\rho_{m}(a, b)$.

Lemma 9. There exists a positive constant $A$ such that for any a, $u$ in $\mathbb{C}^{n}$ with $|a|>A$ and $|u||a|^{\frac{2}{\alpha}-1}<1$, we have

$$
\frac{F\left(|a+u|^{2}\right)}{e^{|a|^{\frac{2}{\alpha}}}|a|^{\frac{1}{\alpha}(1-\alpha)(2 n)}} \approx\left[1+|u||a|^{\frac{2}{\alpha}-1}\right] .
$$

Proof. A Taylor's exansion formula gives

$$
\begin{aligned}
F\left(|a+u|^{2}\right) & =F\left(|a|^{2}\right) \\
& +\int_{0}^{1} F^{\prime}\left(|a|^{2}+t\left(\langle u, a\rangle+\langle a, u\rangle+|u|^{2}\right) d t \times\left(\langle u, a\rangle+\langle a, u\rangle+|u|^{2}\right)\right.
\end{aligned}
$$

By Lemma 6, there are positive constants $A_{4}, C_{4}, C_{5}$ such that for $a, u$ in $\mathbb{C}^{n}$ with $|a|>A_{4}$ and $|u|<1$, we have

$$
\left|F\left(|a+u|^{2}\right)-F\left(|a|^{2}\right)\right| \leq C_{4}|u||a| e^{|a|^{\frac{2}{\alpha}}}|a|^{\frac{2}{\alpha}(1-\alpha)(n+1),}
$$

and

$$
\left(1-C_{5}\right) e^{|a|^{\frac{2}{\alpha}}}|a|^{\frac{1}{\alpha}(1-\alpha) 2 n} \leq\left|F\left(|a|^{2}\right)\right| \leq\left(1+C_{5}\right) e^{|a|^{\frac{2}{\alpha}}}|a|^{\frac{1}{\alpha}(1-\alpha) 2 n}
$$


Therefore

$1-C_{5}-C_{4}|u \|||||a|^{\frac{2}{\alpha}(1-\alpha)} \leq \frac{F\left(|a+u|^{2}\right)}{e^{|a|^{\frac{2}{\alpha}}}|a|^{\frac{2}{\alpha}(1-\alpha) n}} \leq 1+C_{5}+C_{4}|u||a||a|^{\frac{2}{\alpha}(1-\alpha)}$.

This completes the proof.

\section{Proofs of the mains results}

Proof of Theorem 1. By Lemma 1, it is sufficient to estimate $\rho_{m}(a, a+u)$, when $a$ and $u$ are in $\mathbb{C}^{n}$. Setting $Z_{0}=|a|^{\frac{2}{\alpha}}$ and applying Lemma 6 , there exist two functions $\epsilon_{1}$ and $\epsilon_{2}$ such that

$$
\left(F F^{\prime}\right)\left(|a|^{2}\right)=e^{2 Z_{0}} Z_{0}^{-(2 n+1) \alpha} P_{n}\left(Z_{0}\right) P_{n+1}\left(Z_{0}\right)\left(1+\epsilon_{1}\left(|a|^{2}\right)\right)
$$

and

$$
\left(F F^{\prime \prime}-F^{\prime 2}\right)\left(|a|^{2}\right)=e^{2 Z_{0}} Z_{0}^{-(2 n+2) \alpha}\left(P_{n} P_{n+2}-P_{n+1}^{2}\right)\left(Z_{0}\right)\left(1+\epsilon_{2}\left(|a|^{2}\right)\right),
$$

where the polynomials $P_{p}$ are defined by (3.2) and

$$
\lim _{t \rightarrow+\infty} \epsilon_{1}(t)=\lim _{t \rightarrow+\infty} \epsilon_{2}(t)=0
$$

The properties of the polynomials $P_{p}$ shown in Lemma 7 imply that

$$
\left(F F^{\prime \prime}-F^{\prime 2}\right)\left(|a|^{2}\right)=\frac{1}{\alpha^{2 n+1}}\left(\frac{1}{\alpha}-1\right) e^{2 Z_{0}} Z_{0}^{-\alpha} Z_{0}^{(2 n+1)(1-\alpha)}\left(1+\epsilon_{3}\left(|a|^{2}\right)\right)
$$

where $\epsilon_{3}$ is a function satisfying $\lim _{t \rightarrow+\infty} \epsilon_{3}(t)=0$. Using the above estimates, Cauchy-Schwarz inequality and Lemma 8 , we see that there exist positive constants $C_{1}, A_{1}$ such that for $a, u$ in $\mathbb{C}^{n}$ with $|a|>A_{1}$, and $|u|<1$ we have

$$
\begin{array}{r}
N(a, a+u) \leq C_{1} e^{2|a|^{\frac{2}{\alpha}}}|a|^{\frac{2}{\alpha}(1-\alpha) 2 n\left[|a|^{\frac{2}{\alpha}-2}\right.}+\left(|a|^{\frac{4}{\alpha}-3}+|a|^{\frac{6}{\alpha}-3}+|a|^{\frac{8}{\alpha}-4}\right. \\
\left.\left.+|a|^{\frac{10}{\alpha}-5}+|a|^{\frac{12}{\alpha}-6}\right)|u|\right]|u|^{2}
\end{array}
$$

By Lemma 9, there exist positive constants $C_{2}, A_{2}$ such that for any $a, u$ in $\mathbb{C}^{n}$ with $|a|>A$ and $|u||a|^{\frac{2}{\alpha}-1}<1$, we have

$$
\frac{1}{D(a, u)} \leq C_{2} \frac{1}{1-|u||a|^{\frac{2}{\alpha}-1}} e^{-2|a|^{\frac{2}{\alpha}}}|a|^{\frac{-2}{\alpha}(1-\alpha) 2 n} \text {. }
$$


Therefore there exist constants $C_{3}$ and $\delta(|a|)>0$ such that if $|u|<\delta(|a|)$, $\rho_{m}(a, a+u)^{2} \leq C_{3}|a|^{\frac{2}{\alpha}-2}|u|^{2}$. By Lemma 1 , the proof is complete.

In the case $n=1$, we can give a more precise estimate for $\rho_{m}(a, a+u)$.

Proof of Theorem 2. Assume $n=1$. With the notation of the previous proof, we get

$$
\left(F F^{\prime}\right)\left(|a|^{2}\right)=\frac{1}{\alpha^{3}} e^{2 Z_{0}} Z_{0}^{3(1-\alpha)}\left(1+\epsilon_{4}\left(|a|^{2}\right)\right)
$$

and

$$
\left(F F^{\prime \prime}-F^{2}\right)\left(|a|^{2}\right)=\frac{1}{\alpha^{3}}\left(\frac{1}{\alpha}-1\right) e^{2 Z_{0}} Z_{0}^{-\alpha} Z_{0}^{3(1-\alpha)}\left(1+\epsilon_{5}\left(|a|^{2}\right)\right),
$$

where the functions $\epsilon_{4}$ and $\epsilon_{5}$ satisfy $\lim _{t \rightarrow+\infty} \epsilon_{4}(t)=\lim _{t \rightarrow+\infty} \epsilon_{5}(t)=0$.

By Lemma 9 there exist positive constants $A_{4}, C_{2}, C_{2}^{\prime}$ such that for any $a, u$ in $\mathbb{C}^{n}$ with $|a|>A_{4}$ and $|u||a|^{\frac{2}{\alpha}-1}<1$, we have

$$
\begin{aligned}
& \frac{1}{2}\left[\frac{1}{\alpha^{4}} e^{2 Z_{0}} Z_{0}^{3(1-\alpha)}|u|^{2}+R(a, u)\right] \leq N(a, u) \\
& \leq \frac{3}{2}\left[\frac{1}{2 \alpha^{4}} e^{2 Z_{0}} Z_{0}^{3(1-\alpha)}|u|^{2}+R(a, u)\right] \\
&|R(a, u)| \leq \quad C_{2} e^{2|a|^{\frac{2}{\alpha}}}|a|^{\frac{2}{\alpha}(1-\alpha) 2}\left[|a|^{\frac{4}{\alpha}-3}+|a|^{\frac{6}{\alpha}-3}\right. \\
&\left.+|a|^{\frac{8}{\alpha}-4}+|a|^{\frac{10}{\alpha}-5}+|a|^{\frac{12}{\alpha}-6}\right]|u|^{3}
\end{aligned}
$$

and

$$
\begin{aligned}
\frac{C_{2}^{\prime}}{1+|u||a|^{\frac{2}{\alpha}-1}} e^{-2|a|^{\frac{2}{\alpha}}}|a|^{\frac{-2}{\alpha}(1-\alpha) 2} & \leq \frac{1}{D(a, u)} \\
& \leq C_{2} \frac{1}{1-|u||a|^{\frac{2}{\alpha}-1}} e^{-2|a|^{\frac{2}{\alpha}}}|a|^{\frac{-2}{\alpha}(1-\alpha) 2} .
\end{aligned}
$$

Therefore

$$
\begin{array}{r}
\frac{N(a, u)}{D(a, u)} \leq \frac{3}{2}\left[\frac{C_{2}}{\alpha^{4}} Z_{0}^{1-\alpha}+C_{2}^{2}\left(|a|^{\frac{4}{\alpha}-3}+|a|^{\frac{6}{\alpha}-3}+|a|^{\frac{8}{\alpha}-4}\right.\right. \\
\left.\left.+|a|^{\frac{10}{\alpha}-5}+|a|^{\frac{12}{\alpha}-6}\right)|u|\right] \frac{|u|^{2}}{1-|u||a|^{\frac{2}{\alpha}-1}}
\end{array}
$$


We have also

$$
\begin{array}{r}
\frac{N(a, u)}{D(a, u)} \geq \frac{1}{2}\left[\frac{C_{2}^{\prime}}{\alpha^{4}} Z_{0}^{1-\alpha}-C_{2}^{\prime 2}\left(|a|^{\frac{4}{\alpha}-3}+|a|^{\frac{6}{\alpha}-3}+|a|^{\frac{8}{\alpha}-4}\right.\right. \\
\left.\left.+|a|^{\frac{10}{\alpha}-5}+|a|^{\frac{12}{\alpha}-6}\right)|u|\right] \frac{|u|^{2}}{1+|u||a|^{\frac{2}{\alpha}-1}} .
\end{array}
$$

So there exist positive numbers $C_{4}, C_{4}^{\prime}$ and $\delta(|a|)$ such that for $|u|<\delta(|a|)$, we have

$$
C_{4}^{\prime} Z_{0}^{1-\alpha}|u|^{2} \leq \frac{N(a, u)}{D(a, u)} \leq C_{4} Z_{0}^{1-\alpha}|u|^{2},
$$

which completes the proof.

We now prove Corollary 3 .

Proof of Corollary 3. Assume $n=1$ and $\frac{1}{\alpha}=r$ is a positive integer; the function $z \mapsto z^{r}$ is analytic in the whole complex plane. The Taylor formula completes the proof.

In the special case when $n=1$ and $m \leq 1$, we shall prove Corollary 4 .

Proof of Corollary 4. Assume $\alpha \geq 2$. Then, in the proof of Theorem 2, the terms $Z_{0}^{1-\alpha},\left[|a|^{\frac{4}{\alpha}-3}+|a|^{\frac{6}{\alpha}-3}+|a|^{\frac{8}{\alpha}-4}+|a|^{\frac{10}{\alpha}-5}+|a|^{\frac{12}{\alpha}-6}\right]$ and $|a|^{\frac{2}{\alpha}-1}$ are uniformly bounded when $|a|>A_{4}$.

\section{The range of $\mathrm{Ber}$}

The Berezin transform Ber : $X \mapsto \tilde{X}$, defined for an operator on $\mathcal{A}^{2}\left(\mu_{m}\right)$ such that both domains of $X$ and $\widetilde{X}$ contain the set $\left\{k_{a}, \in \mathbb{C}^{n}\right\}$ is linear and one-to-one (see ). If $O p$ denotes the algebra of all linear bounded operators on $\mathcal{A}^{2}\left(\mu_{m}\right)$, we showed that $\operatorname{Ber}(O p)$ is a linear subspace of locally Lipschitz functions on $\mathbb{C}^{n}$. As in the classical case (see [5]), we can show that smooth bounded Lipschitz functions can fail to be in $\operatorname{Ber}(O p)$.

Theorem 5. Assunme $m>0$. There is no $X$ in $O p$ such that $\tilde{X}(a)=\frac{K_{m}(t a, a)}{K_{m}(a, a)}$, where $K_{m}$ is the reproducing kernel of $\mathcal{A}^{2}\left(\mu_{m}\right)$ and $t \in]-\infty,-1\left[,|t|^{\frac{1}{\alpha}} \cos \frac{\pi}{\alpha}<1\right.$.

Proof. We use the notation $K$ instead of $K_{m}$ and $d \mu$ instead of $d \mu_{m}$. For a real number $t$, let $R_{t}$ be the linear operator on $\mathcal{A}^{2}\left(\mu_{m}\right)$ defined by $R_{t}(f)(z):=f(t z)$. First, we consider the boundedness of $R_{t}$. Using the 
properties of the reproducing kernel, we compute

$$
\left\|R_{t} k_{a}\right\|^{2}=\frac{1}{K(a, a)} K(a t, a t)
$$

Observe that $\left\|R_{t} k_{a}\right\|^{2} \leq 1$ for all $|t| \leq 1$. On the other hand, if $|t|>1$, we have

$$
\left\|R_{t} k_{a}\right\|^{2} \sim e^{|a|^{\frac{2}{\alpha}}\left(|t|^{\frac{2}{\alpha}}-1\right)}|t|^{\frac{2 n}{\alpha}(1-\alpha)}, \text { when }|a| \rightarrow+\infty .
$$

Therefore $\lim _{|a| \rightarrow+\infty}\left\|R_{t} k_{a}\right\|^{2}=+\infty$ and hence $R_{t}$ is unbounded. The Berezin tranform of $R_{t}$ is given by

$$
\widetilde{R}_{t}(a)=\left\langle R_{t} k_{a}, k_{a}\right\rangle=\frac{1}{K(a, a)} K(a t, a) .
$$

We are going to show that there is no bounded operator $X$ on $\mathcal{A}^{2}\left(\mu_{m}\right)$ such that

$$
\widetilde{X}(a)=\frac{K(t a, a)}{K(a, a)}
$$

We observe that the function $g_{t}(a)=\frac{K(t a, a)}{K(a, a)}$ is real-differentiable at every order. Assume that $t<0$. We will show that $g$ and its first order derivatives are bounded on $\mathbb{C}^{n}$. We have

$$
g_{t}(a)=\frac{F\left(t|a|^{2}\right)}{F\left(|a|^{2}\right)}
$$

and

$$
\frac{\partial g}{\partial a_{j}}(a)=\bar{a}_{j}\left[\frac{t F^{\prime}\left(t|a|^{2}\right) F\left(|a|^{2}\right)-F^{\prime}\left(t|a|^{2}\right)}{F^{2}\left(|a|^{2}\right)}\right] .
$$

With the notations of Section 2, for $z \in \mathbb{C} \backslash\{0\}$, we have the asymptotic expansions

$$
\begin{gathered}
F(z)=\sum_{s \in \mathcal{S}} \frac{e^{Z_{s}}}{z^{n}} P_{n}\left(Z_{s}\right)+\widetilde{\mathcal{R}}^{(n-1)}(z), \\
F^{\prime}(z)=\sum_{s \in \mathcal{S}} \frac{e^{Z_{s}}}{z^{n+1}} P_{n+1}\left(Z_{s}\right)+\widetilde{\mathcal{R}}^{(n)}(z),
\end{gathered}
$$

where $Z_{s}=|z|^{\frac{1}{\alpha}} \exp \left[\frac{i \pi}{\alpha}(1+2 s)\right]$, for s integer, and for $p \in\{n-1, n\}$,

$$
\widetilde{\mathcal{R}}^{p}(z)=\sum_{k=0}^{N-p}(-1)^{p+1} \frac{(k+p) !}{p !} \frac{1}{\Gamma(-k \alpha)} \frac{1}{z^{p+1+k}}+\mathcal{R}_{N}^{(p)}(z) .
$$


First consider $F\left(t|a|^{2}\right)$. Setting $z=t|a|^{2}$ and $Z_{+}=e^{\frac{i \pi}{\alpha}}|t|^{\frac{1}{\alpha}}|a|^{\frac{2}{\alpha}}$ and $Z_{-}=$ $e^{\frac{-i \pi}{\alpha}}|t|^{\frac{1}{\alpha}}|a|^{\frac{2}{\alpha}}$, we have $\arg z=\pi$, and hence

$$
F(z)=\frac{1}{z^{n}}\left[e^{Z_{+}} P_{n}\left(Z_{+}\right)+e^{Z_{-}} P_{n}\left(Z_{-}\right)\right]+\rho_{0, \pi}(z)
$$

and

$$
F^{\prime}(z)=\frac{1}{z^{n+1}}\left[e^{Z_{+}} P_{n+1}\left(Z_{+}\right)+e^{Z_{-}} P_{n+1}\left(Z_{-}\right)\right]+\rho_{1, \pi}(z)
$$

with $\rho_{0, \pi}(z)=o\left(e^{Z_{+}} Z_{+}^{n(1-\alpha)}\right)$ and $\rho_{1, \pi}(z)=o\left(e^{Z_{+}} Z_{+}^{(n+1)(1-\alpha)}\right)$ when $|z| \rightarrow+\infty$.

Also, when $|a| \rightarrow+\infty$, setting $Z_{0}=|a|^{\frac{2}{\alpha}}$ we obtain

$$
F\left(|a|^{2}\right)=\frac{1}{|a|^{2 n}} e^{Z_{0}} P_{n}\left(Z_{0}\right)+\rho_{0,0}\left(|a|^{2}\right),
$$

and

$$
F^{\prime}\left(|a|^{2}\right)=\frac{1}{|a|^{2 n+2}} e^{Z_{0}} P_{n+1}\left(Z_{0}\right)+\rho_{1,0}\left(|a|^{2}\right),
$$

where $\rho_{j, 0}\left(|a|^{2}\right)=o\left(e^{|a|^{\frac{2}{\alpha}}}|a|^{\frac{2}{\alpha}(n+j)(1-\alpha)}\right), j=0,1$. Now we compute $N_{t}(a)=t F^{\prime}\left(t|a|^{2}\right) F\left(|a|^{2}\right)-F^{\prime}\left(|a|^{2}\right) F\left(t|a|^{2}\right)$. The main term in $N_{t}(a)$ is

$$
\begin{array}{r}
\widetilde{N_{t}(a)}=\frac{1}{|t|^{n}|a|^{2(2 n+1)}}\left\{e^{Z_{0}+Z_{+}}\left[P_{n}\left(Z_{0}\right) P_{n+1}\left(Z_{+}\right)-P_{n+1}\left(Z_{0}\right) P_{n}\left(Z_{+}\right)\right]\right. \\
\left.+e^{Z_{0}+Z_{-}}\left[P_{n}\left(Z_{0}\right) P_{n+1}\left(Z_{-}\right)-P_{n+1}\left(Z_{0}\right) P_{n}\left(Z_{-}\right)\right]\right\}
\end{array}
$$

Thus

$$
\widetilde{N_{t}(a)} \sim \frac{Z_{0}^{n} e^{Z_{0}}}{\alpha^{2 n+1}|t|^{n}|a|^{2(2 n+1)}} 2 \Re\left\{e^{Z_{+}} Z_{+}^{n}\left(Z_{+}-Z_{0}\right)\right\} .
$$

Observing that

$$
\left|e^{Z_{+}} Z_{+}^{n}\left(Z_{+}-Z_{0}\right)\right|=\left.\exp \left[\cos \frac{\pi}{\alpha}|t|^{\frac{1}{\alpha}}|a|^{\frac{2}{\alpha}}\right]|t|^{\frac{n}{\alpha}}|a|^{\frac{2 n+2}{\alpha}}|| e^{i \frac{\pi}{\alpha}} t\right|^{\frac{1}{\alpha}}-1 \mid,
$$

we obtain an estimation of $N_{t}(a)$; there exist positive constants $A, C$ such that for $a \in \mathbb{C}^{n}$ with $|a|>A$,

$$
\left|N_{t}(a)\right| \leq\left. C|t|^{n\left(\frac{1}{\alpha}-1\right)}\left|e^{i \frac{\pi}{\alpha}}\right| t\right|^{\frac{1}{\alpha}}-\left.1|| a\right|^{(4 n+2)\left(\frac{1}{\alpha}-1\right)} \exp \left[\left(1+|t|^{\frac{1}{\alpha}} \cos \frac{\pi}{\alpha}\right)|a|^{\frac{2}{\alpha}}\right] .
$$

On the other hand, we have,

$$
F\left(|a|^{2}\right) \sim \frac{1}{\alpha^{n}}|a|^{2 n\left(\frac{1}{\alpha}-1\right)} \exp |a|^{\frac{2}{\alpha}},|a| \rightarrow+\infty .
$$


Therefore there exist positive constant $A^{\prime}, C^{\prime}$ such that for any $j \in[1, n]$, if $|a|>A^{\prime}$,

$$
\begin{gathered}
\left|g_{t}(a)\right| \leq C^{\prime} \exp \left[\left(-1+|t|^{\frac{1}{\alpha}} \cos \frac{\pi}{\alpha}\right)|a|^{\frac{2}{\alpha}}\right], \\
\left|\frac{\partial g_{t}}{\partial a_{j}}(a)\right| \leq C^{\prime}|a||a|^{2\left(\frac{2}{\alpha}-1\right)} \exp \left[\left(-1+|t|^{\frac{1}{\alpha}} \cos \frac{\pi}{\alpha}\right)|a|^{\frac{2}{\alpha}}\right] .
\end{gathered}
$$

Chosing $t \in]-\infty,-1\left[\right.$ and $|t|^{\frac{1}{\alpha}} \cos \frac{\pi}{\alpha}<1$, we see that $g_{t}$ and its derivatives of the first order are bounded; in particular $g_{t}$ is Lipschitz.

Suppose there exist a bounded operator $X$ in $O p$ with $\widetilde{X}(a)=g_{t}(a)$. The operator $A:=X-R_{t}$ is in $O p$ and satisfies $\widetilde{A}(a)=0$. The injectivity of the mapping $A \mapsto \widetilde{A}[7]$ leads to $A=0$. Then $X k_{a}=R_{t} k_{a}$, which contradicts the boundedness of $X$.

Acknowledgement. I wish to thank El Hassan Youssfi for useful discussions.

\section{References}

[1] H. Bommier and E. H. Youssfi, Hankel operators on weighted Fock spaces, Integr. Equ. Oper. Theory, 59 (2007) 1-17.

[2] C.A. Berger and L.A. Coburn, Toeplitz Operators on the SegalBargmann space, Trans. Amer. Math . Soc., 301 (1987), 813-829.

[3] C.A. Berestein and R. Gay, Complex Analysis and Special Topics in Harmonic Analysis, Springer, 1995.

[4] L.A. Coburn, A Lipschitz estimate for Berezin's operator calculus, Proc. Amer. Math. Soc., 133 (2005), 127-131.

[5] L.A. Coburn, Sharp Berezin Lipschitz estimates, Proc. Amer. Math. Soc., 135 (2005), 1163-1168.

[6] M. Englis, Berezin transforms on Pluriharmonic Bergman spaces, preprint.

[7] M. Englis, Singular Berezin transforms, preprint.

[8] M. Englis and G. Zhang On the derivatives of the Berezin transform, Proc. Amer. Math. Soc., 134 (2006), 2285-2294.

[9] M. Jarnicki and P. Pflug, Invariant distances and metrics in complex analysis, Walter de Gruyter, 1993.

[10] R. Wong and Y.Q. Zhao, Exponential asymptotics of the Mittag-Leffler function, Constr. Approx., 18 (2002), 355-385. 
LATP, U.M.R. C.N.R.S. 6632, CMI

Université De Provence

39 Rue Fjoliot-Curie

13453 Marseille Cedex 13

France

(E-mail : bommier@cmi.univ-mrs.fr)

(Received : November 2007) 


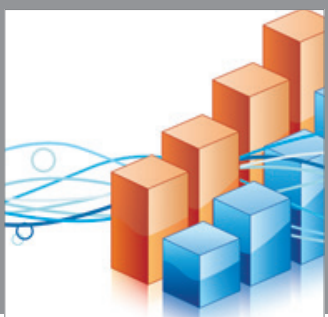

Advances in

Operations Research

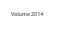

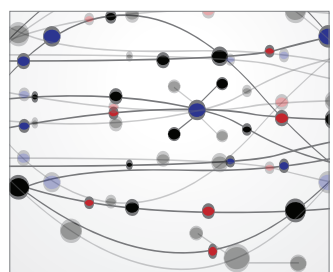

\section{The Scientific} World Journal
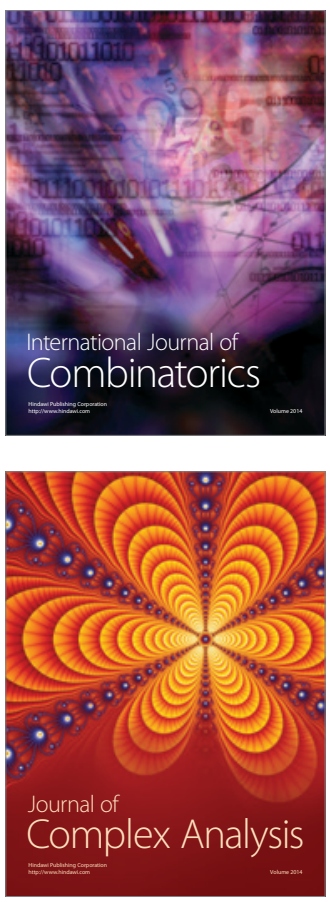

International Journal of

Mathematics and

Mathematical

Sciences
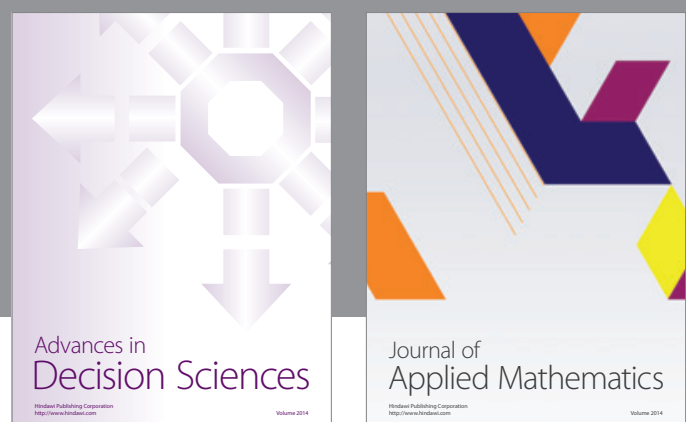

Journal of

Applied Mathematics
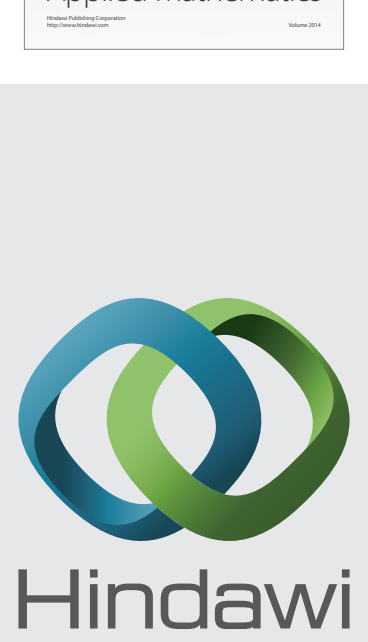

Submit your manuscripts at http://www.hindawi.com
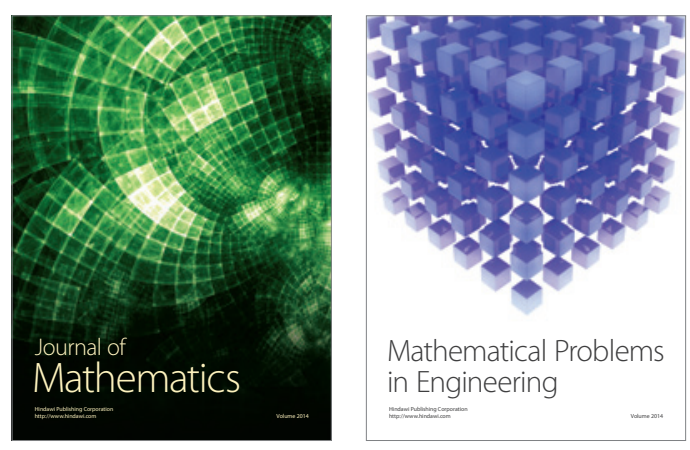

Mathematical Problems in Engineering
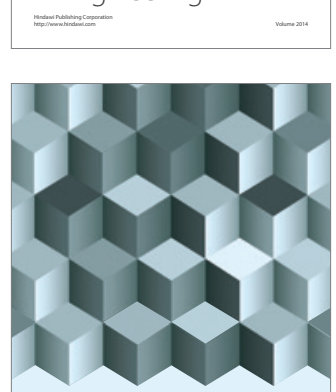

Journal of

Function Spaces
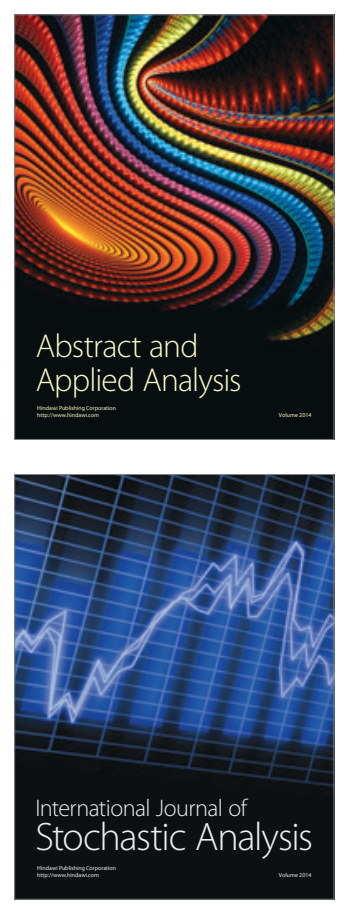

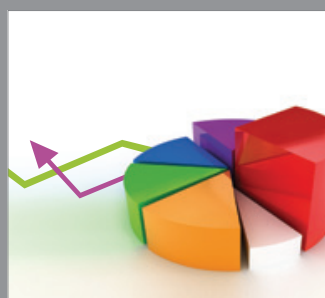

ournal of

Probability and Statistics

Promensencen
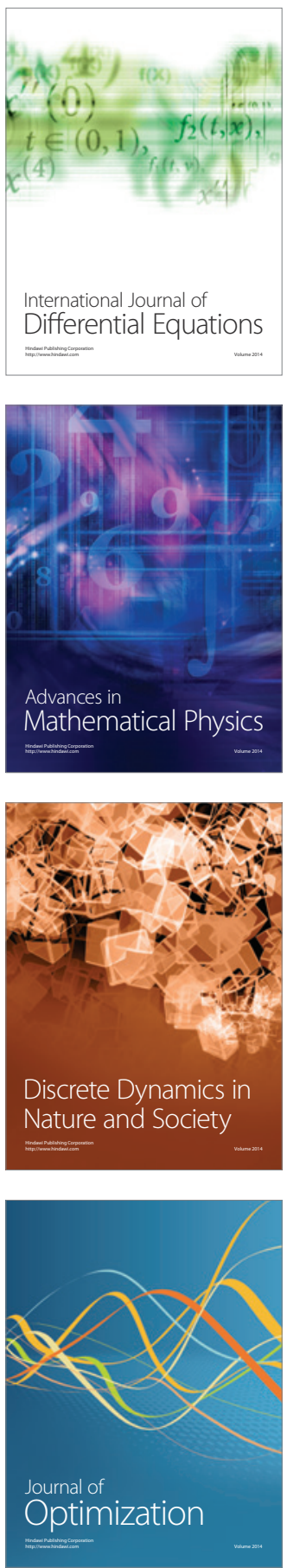